Aladro, A. Capitalismo, patriarcado y ciudad: generalidades de la producción de lo urbano desde una perspectiva de género. Derecho y Ciencias Sociales. Mayo- Octubre $2019 \mathrm{~N}^{\circ} 21$. (Derecho, ciudad y propiedad) Pgs 23-36. ISNN 1852-2971. Instituto de Cultura Jurídica y Maestría en Sociología Jurídica. FCJ y S. UNLP

\title{
Capitalismo, patriarcado y ciudad: generalidades de la producción de lo urbano desde una perspectiva de género
}

Capitalism, patriarchy and city: generalities of urban production from a gender perspective

Almendra Aladro

\section{Resumen}

El presente artículo se propone analizar la diferenciación conceptual entre los procesos de producción y reproducción en esta etapa de desarrollo del modelo capitalista desde una perspectiva de género que permita comprender cómo el proceso de producción del espacio urbano excluye históricamente a las mujeres en cuanto al acceso a la ciudad como derecho en los términos explicados por Ana Falú e intentará, en consecuencia, reconstruir un posible marco metodológico que facilite la recuperación de la vida cotidiana de las mujeres como experiencia política situada en los términos de Henri Lefebvre (1968). Para ello, se echará mano a obras pertenecientes tanto al campo del Urbanismo, como de la Filosofía Política, la Sociología y el Derecho, para generar un diálogo de saberes para el abordaje de tan complejo objeto de estudio.

Palabras clave: derecho a la ciudad, género, etnografía, políticas públicas, políticas locales

\begin{abstract}
This article aims to analyze the conceptual differentiation between the processes of production and reproduction in this stage of development of the capitalist model from a gender perspective that allows to understand how the production process of urban space historically excludes women in terms of access to the city as a right in the terms explained by Ana Falú and will try, consequently, to reconstruct a possible methodological framework that facilitates the recovery of the daily life of women as a political experience located in the terms of Henri Lefebvre (1968). To do this, works will be taken up belonging to the field of Urbanism, as well as Political Philosophy, Sociology and Law, proposing a dialogue of knowledge for the approach of such a complex object of study.
\end{abstract}

Keywords: right to the city, gender, ethnography, public policies, local policies

\footnotetext{
- Abogada por la Universidad Nacional de Mar del Plata (FD-UNMdP). Becaria doctoral de CONICET con lugar de trabajo en el Centro de Investigación y Docencia en Derechos Humanos "Dra. Alicia Moreau" (CIC-UNMdP). aaladro@mdp.edu.ar; ORCID: https://orcid.org/0000-0003-2658-6794/
}

Recibido: 28/2/2019. Publicable con correcciones: 6/9/2019. 
Aladro, A. Capitalismo, patriarcado y ciudad: generalidades de la producción de lo urbano desde una perspectiva de género. Derecho y Ciencias Sociales. Mayo- Octubre 2019 N² 21. (Derecho, ciudad y propiedad) Pgs 23-36. ISNN 1852-2971. Instituto de Cultura Jurídica y Maestría en Sociología Jurídica. FCJ y S. UNLP

\section{Capitalismo, patriarcado y ciudad: generalidades de la producción de lo urbano desde una perspectiva de género}

Almendra Aladro

\section{Introducción}

La descripción del rol de los espacios urbanos en la lucha de clases fue abordada por Henri Lefebvre, sociólogo que indaga en el proceso de urbanización como fenómeno diferente y específico pero estrechamente vinculado a la industrialización que describieran Marx y Engels en su obra. Reconociendo la preexistencia de las ciudades respecto de las fábricas, el autor realiza una lectura crítica de la obra marxista en la cual encuentra una imposibilidad para describir la estrecha relación entre la producción del espacio urbano y la producción industrial, identificando a las ciudades como el lugar en el cual la mano de obra asalariada se agrupa, donde se introducen las mercancías y el punto desde el que se dirigen los negocios (Lefebvre, 1968). En este mismo sentido, Lombardo (2012) afirma que existe una articulación entre "la construcción del espacio, el proceso de producción y circulación del capital, la reproducción de la fuerza de trabajo y del capital, y las contradicciones derivadas de la base en que se asienta la apropiación del trabajo" (Lombardo, 2012:30) y, desde la base teórica sistematizada por David Harvey (2006) sostiene que la formación y transformación del espacio geográfico constituye una expresión de las contradicciones propias del modo de producción capitalista.

La obra de Lombardo gira en torno a lo que denomina "reproducción social de la vida en un territorio" entendiendo por tal al proceso de construcción de condiciones materiales e inmateriales desarrollado por los actores sociales presentes en un territorio y que tiene por objetivo sostener el desarrollo de su existencia en dicho espacio, resultando así una construcción socioespacial de carácter sistémica (Lombardo, 2012). Para desarrollar conceptualmente esta idea, Lombardo toma la afirmación de Carlos (2008) en cuanto a que el espacio geográfico es "condición, medio y producto de la producción de la sociedad" (Carlos, 2008:s/n) y que la reproducción del capital como proceso articula la totalidad de las dimensiones de una formación social, por lo cual el individuo no debe ser sólo pensado como fuerza de trabajo, sino también como un sujeto en el proceso de producción del espacio (Carlos, 2008). 
Ana Falú (1998) suma a este desarrollo una perspectiva que busca evidenciar la omisión de las mujeres $^{1}$ en la forma tradicional de pensar las ciudades, explicando que ellas permanecerán invisibilizadas en los espacios urbanos como consecuencia de la división sexual del trabajo, que vincula al hombre con el trabajo productivo y a las mujeres con la responsabilidad del trabajo doméstico y reproductivo. A partir de esta afirmación, se posiciona políticamente demandando la visibilización de dicha circunstancia frente al uso de conceptos que se presentan como "neutrales" y a partir de los cuales las ciudades son planificadas sin pensar a las mujeres como ciudadanas y titulares de derechos (Falú, 2016). La división del espacio derivada de las actividades productivas frente a las reproductivas ha sido abordada por Carole Pateman (1995) en su crítica a la dicotomía público/privado instalada por el liberalismo lockeano y es un aporte desde la filosofía política a observar al momento de intentar dar cuenta de la situación de las mujeres en contextos urbanos dentro del modo de producción capitalista.

Es decir, que la (re)ocupación de los espacios públicos por parte de las mujeres con el objetivo de llevar adelante la lucha por la liberación de la opresión masculina y heterosexual, tiene, al menos, dos formas de ser observada. En primer lugar, como una conquista del espacio público en el sentido en que lo critica Pateman y, en segundo lugar, como una exigencia de transformación de las ciudades en espacios producidos sin jerarquización de los roles sociales basada en el género.

El presente artículo se propone analizar la diferenciación conceptual entre los procesos de producción y reproducción en esta etapa de desarrollo del sistema capitalista desde una perspectiva de género que permita comprender cómo el proceso de producción del espacio urbano excluye históricamente a las mujeres en cuanto al acceso a la ciudad como derecho en los términos explicados por Ana Falú. Asimismo intentará, en consecuencia, reconstruir un posible marco metodológico que facilite la recuperación de la vida cotidiana de las mujeres como experiencia política situada en los términos de Henri Lefebvre (1968). Para ello, se echará mano a obras pertenecientes tanto al campo del Urbanismo, como de la Filosofía Política, la Sociología y el Derecho, proponiendo un diálogo de saberes para el abordaje de tan complejo objeto de estudio.

\section{Las actividades de producción y reproducción como génesis de la dicotomía "público- privado"}

\footnotetext{
${ }^{1}$ Corresponde aclarar que en este artículo la palabra "mujer" siempre comprenderá a mujeres cisgénero y transgénero.
} 
Los debates en torno a la división del trabajo en productivo y reproductivo se desprenden de una discusión precedente en relación a la existencia del patriarcado como sistema autónomo respecto del capitalismo. Con respecto a este último asunto, si bien excede el objeto de la presentación ahondar en dichas discusiones, en este trabajo se adopta una postura feminista y materialista que afirma que las relaciones sociales estructuradas en base al género se vinculan con la aparición misma de la propiedad privada pero se sofistican al consolidarse el capitalismo, y esa relación de articulación es dialéctica y, por ende, contradictoria. ${ }^{2}$

Partiendo de dicha postura teórica, corresponde ahora adentrar el análisis respecto de los procesos de producción y reproducción en el modo de producción capitalista y su vínculo con las relaciones estructuradas en base al género. Roswitha Scholz (2013) explica esta cuestión, a partir de la Teoría Crítica de Adorno y de los desarrollos de Robert Kurtz y Moishe Postone dentro de la Crítica de la Economía Política de Marx, desde la teoría de la escisión del valor. Así, la vida social se divide en dos esferas jerárquicamente relacionadas: el trabajo productivo y las actividades reproductivas. Explica que "las mujeres han de responsabilizarse ante todo del ámbito de la reproducción, menos valorado socialmente y no representable en dinero, mientras que los hombres se ocupan de la esfera de la producción capitalista y del ámbito público" (Scholz, 2013:49) y esta forma de dinámica social comienza su desarrollo con el modo de producción capitalista, si bien las relaciones de género encuentran su origen en una etapa precapitalista (Engels, 2011). Entiende que el modo de producción capitalista en su fase actual "tiene su condición de posibilidad en las mujeres, así como en una postergación de lo social y de la naturaleza" (Scholz, 2013:50) e identifica "dicotomías típicas y oposiciones antagónicas del patriarcado productor de mercancías" (Scholz, 2013:50) como "sujeto-objeto", "espíritunaturaleza", “dominación-sometimiento", "varón-mujer”, "público-privado".

Sobre la última díada enumerada, relevante para el objetivo general de este trabajo, realiza una interesante observación Carole Pateman (2009), quien, en su crítica al liberalismo, afirma que existe un sector del feminismo que discute la dicotomía "público-privado" tomando como punto de partida la misma perspectiva que le da origen, la cual identifica en la teoría lockeana, y que termina por sostener no sólo la existencia de un espacio político (público) y uno de tipo doméstico (privado) que constituyen esferas separadas, opuestas pero fuertemente interrelacionadas. La autora formula en su trabajo que el tipo de liberalismo al cual adhiere el

\footnotetext{
${ }^{2}$ Ver: Engels (2011), Federici (2015) y Scholz (2013).
} 
sector del pensamiento feminista ${ }^{3}$ evocado, afirma la existencia de la sociedad civil con prescindencia de la vida en el ámbito doméstico, la cual es además invisibilizada en el debate, y esta idea es también llevada como división al seno de la propia sociedad civil, es decir, del ámbito exclusivo masculino.

Sen (1996) reflexiona sobre las repercusiones en la vida de las mujeres de la dicotomía públicoprivado $\mathrm{y}$, en definitiva, de su origen inmediato (la escisión de las relaciones sociales en actividades productivas y reproductivas) y sostiene que "las mujeres están situadas en la encrucijada entre producción y reproducción (...) entre el crecimiento económico y el desarrollo humano" (Sen, 1996:60).

Hasta aquí se ha pretendido realizar un breve recorrido teórico que, a los fines de este trabajo, se considera elemental para la compresión de los acápites siguientes, en los cuales se buscará generar un diálogo entre autores y autoras que permita explicar cómo esta lógica de la escisión de la vida social en productiva y reproductiva se traslada a la producción de bienes urbanos, lo cual tiene como resultado la falta de acceso de las mujeres a la ciudad como derecho, y relevar sus prácticas urbanas desde un punto de vista político para el diseño de políticas públicas que aborden la cuestión con una perspectiva de género.

\section{El derecho a la ciudad desde una perspectiva de género}

El hito iniciático en la elaboración del concepto de derecho a la ciudad es la obra de Henri Lefebvre que, precisamente, se titula "El derecho a la ciudad" y donde sostiene como una de sus premisas centrales que,

Lo urbano (...) es el resultado de una historia, de una praxis social, de personas y grupos determinados que realizan esta obra en condiciones históricas (...)” y, así, “(...)

\footnotetext{
${ }^{3}$ Carole Pateman ha dedicado gran parte de su obra a la crítica del liberalismo lockeano en general y al feminismo liberal del siglo XIX en particular. Si bien excede el motivo de este trabajo entrar en tal debate, cabe decir que la autora en el prefacio de El contrato sexual (1995) explica sucintamente su tesis central al respecto:

El contrato sexual es una dimensión reprimida de la teoría del contrato, una parte integral de la elección racional del familiar acuerdo original. El contrato originario, tal como se debería entender hoy, es sólo una parte del acto de la génesis política descrito en las páginas de los teóricos clásicos del contrato de los siglos XVII y XVIII. El objetivo de mi estudio es comenzar a abrir la censura en los estratos teóricos sobre la que descansa (Pateman, 1995:1).

A partir de allí, Pateman desarrolla los puntos en común y las diferencias entre feminismo liberal y liberalismo lockeano, haciendo énfasis en cómo la aceptación acrítica de ese liberalismo implica asumir compromisos teóricos difíciles de defender por quienes se consideran feministas liberales.
} 
Aladro, A. Capitalismo, patriarcado y ciudad: generalidades de la producción de lo urbano desde una perspectiva de género. Derecho y Ciencias Sociales. Mayo- Octubre $2019 \mathrm{~N}^{\circ} 21$. (Derecho, ciudad y propiedad) Pgs 23-36. ISNN 1852-2971. Instituto de Cultura Jurídica y Maestría en Sociología Jurídica. FCJ y S. UNLP

la ciudad como obra, como resultado de esta praxis, así la vida cotidiana se torna obra, apropiación del espacio, uso. (Lefebvre, 1967:68)

Desde el materialismo dialéctico los escritos de Lefebvre analizan las ciudades y la urbanización como espacios de desenvolvimiento de la lucha de clases y cuya producción no escapa a la lógica de la producción de mercancías. A partir de las ideas de Lefebvre, otros autores han realizado críticas, relecturas y redefiniciones del concepto de derecho a la ciudad. Así, Harvey (2012) profundiza la idea de que el derecho a la ciudad es un derecho colectivo de todas las personas que habitan, acceden y usan la ciudad y supone no solamente el derecho a usar aquello que ya existe, sino también a definir y crear lo que debería existir en los espacios urbanos en miras a la satisfacción de la necesidad de llevar una vida digna y plena en dicho entorno.

Jordi Borja (2001) aporta una dimensión jurídica de esta construcción político-filosófica, refiriendo a la idea de "construir ciudad" en el siglo XXI como una política que proyecte ejercicio de la ciudadanía, ampliación de los denominados "derechos de tercera generación" (derechos económicos, sociales, culturales y ambientales), el "derecho al lugar y a la movilidad, a la ciudad refugio y a la identidad local, al autogobierno y a la diferencia, a la igualdad jurídica de todos los residentes y a la proyección exterior” (Borja, 2001:395).

Edward Soja (2014), por su parte, incorpora a la idea de ciudad como espacio de la lucha de clases propuesta por Lefebvre y su recuperación a través de prácticas insurgentes que vehiculicen la participación de la ciudadanía en la construcción de los espacios urbanos esbozada por Harvey, la noción de "justicia espacial", a partir de la cual el autor critica las posturas marxistas tradicionales respecto a la ciudad por excluir a través del concepto de lucha de clases, perspectivas como el género, las migraciones, las cuestiones étnicas y las culturales, aristas clave al momento del análisis de la construcción y prácticas urbanas. Metodológicamente, el autor propone la "trialéctica" o estudio del tercer espacio, una forma de abordaje de la ciudad como objeto de estudio que contempla tres dimensiones de análisis, a saber: 1) el mundo real y material; 2) las representaciones sociales; y 3) el espacio producido a partir de las dimensiones 1) y 2) (Soja, 2014). A los fines de este trabajo la producción de Edward Soja será considerada como un avance fundamental en la construcción del concepto de derecho a la ciudad, en tanto incorpora las posibles perspectivas y desafíos que implica pensar al mismo desde el punto de vista de los grupos desaventajados, entre los cuales se encuentran las mujeres. 
Aladro, A. Capitalismo, patriarcado y ciudad: generalidades de la producción de lo urbano desde una perspectiva de género. Derecho y Ciencias Sociales. Mayo- Octubre 2019 N² 21. (Derecho, ciudad y propiedad) Pgs 23-36. ISNN 1852-2971. Instituto de Cultura Jurídica y Maestría en Sociología Jurídica. FCJ y S. UNLP

Enseña Ana Falú que "las mujeres permanecerán invisibles en las ciudades, relegadas al ámbito privado del hogar, lo que tendrá sustento en la fuerte división del trabajo sexual imperante" (Falú, 2016:160), afirmación en la que se encuentra latente la dicotomía público/privado como eje estructurante de las desigualdades urbanas basadas en género. En este razonamiento, "las ciudades no son percibidas ni usadas de la misma manera por hombres y mujeres" ya que las mujeres "acceden a los bienes y servicios públicos de manera distinta, combinando trabajo productivo y reproductivo" (Falú, 2016:163). De este uso diferencial de los bienes urbanos y la configuración de prácticas urbanas propias dan cuenta numerosas investigaciones cuanti y cualitativas relativas a la movilidad urbana compiladas por Paola Jirón y Dhan Zunino Singh (2017), dossier del cual se desprende la coincidencia entre distintos investigadores e investigadoras de distintas partes del mundo respecto del protagonismo de las tareas y actividades de cuidado en la configuración del territorio y desplazamiento de las mujeres.

\section{1 "Dimensiones" del derecho a la ciudad en la obra de Ana Falú}

Se entiende aquí que debe señalarse una inquietud respecto de una afirmación de Ana Falú en la que asegura que las mujeres no son "debidamente significadas como sujetos de derecho a la ciudad" (Falú, 2016:163). ¿Qué puede querer decir la autora con esta frase si, al menos en lo que, en general, a occidente refiere, y en particular, a nuestro país corresponde, existen dispositivos que positivizan el derecho a la igualdad ante la ley y la prohibición de discriminación por razones de sexo/género?

La clave para desentrañar la cuestión se encuentra en una pregunta que la misma Falú se hace tras reflexionar sobre la desigualdad y la subordinación de género, sumadas a la división sexual del trabajo, como elementos desatendidos que afectan la planificación de ciudades y vecindarios: “¿Cuánto de esta omisión incide en la posibilidad de acceso y el buen uso de los bienes urbanos?" (Falú, 2016:163).

En el interrogante transcripto pueden identificarse, al menos, dos dimensiones del derecho a la ciudad: por un lado, el acceso a la ciudad y, por el otro, el "buen uso" (que aquí se llamará "goce") de los bienes urbanos. No obstante, Falú no conceptualiza las dimensiones identificadas. Por ello, aquí se comprenderá a la "dimensión de acceso" como las condiciones materiales, sociales, culturales, físicas, biológicas, personales, etc., con las cuales cuenta la persona titular de un derecho y que representan variables que repercuten en la posibilidad de ejercicio real del derecho; mientras que se indicará la "dimensión de goce" como el ejercicio real y completo del derecho en cuestión por parte de la persona titular. 
Aladro, A. Capitalismo, patriarcado y ciudad: generalidades de la producción de lo urbano desde una perspectiva de género. Derecho y Ciencias Sociales. Mayo- Octubre 2019 N² 21. (Derecho, ciudad y propiedad) Pgs 23-36. ISNN 1852-2971. Instituto de Cultura Jurídica y Maestría en Sociología Jurídica. FCJ y S. UNLP

La igualdad ante la ley supone garantizar la no turbación en la dimensión de goce de un derecho en tanto constituye una ficción que presupone que todos y todas han podido acceder al mismo desde las mismas condiciones de base. En el caso de las mujeres, su acceso a la ciudad se ve dificultado desde un juego de condiciones que implica que por ser la ciudad producida desde la lógica del capital, y como espacio público por excelencia, es diseñado desde la visión que el patriarcado capitalista impone respecto de las necesidades de los varones y las mujeres y sus "lugares naturales". Así, la incorporación de las mujeres al mercado laboral y la conquista de derechos progresiva desatada desde entonces genera una contradicción en el modelo patriarcal y capitalista de ciudad y la necesidad de gestionar prácticas urbanas propias, en tanto mundo público y mundo privado, finalmente, son categorías espaciales cuyos límites siempre fueron impuestos desde la propia estructura de dominación.

\section{Discusión: recuperar la dimensión política de la vida cotidiana de las mujeres desde la Etnografía Institucional para romper la dicotomía "público-privado"}

La preocupación por la "vida cotidiana" que Henri Lefebvre expone en sus obras Crítica de la vida cotidiana [1947] (1991), La vida cotidiana en el mundo moderno (1967) e Introducción a la psicosociología de la vida cotidiana (1970) no constituye un capítulo de su trabajo académico, sino que se presenta como el telón de fondo que moviliza su compleja labor científica (Lindón Villoría, 2004). Lefebvre refiere a lo largo de su obra de una suerte de fases o dimensiones de la idea de "lo cotidiano":

En su trivialidad, lo cotidiano se compone de repeticiones: gestos en el trabajo y fuera del trabajo, movimientos mecánicos (los de las manos y los del cuerpo, y también los de las piezas y los dispositivos, rotación o ida y vuelta), horas, días, semanas, meses, años; repeticiones lineales, y repeticiones cíclicas, tiempo de la naturaleza y tiempo de la racionalidad, etcétera. El estudio de la actividad creadora (de la producción en el sentido más amplio) conduce hacia el análisis de la reproducción, es decir, de las condiciones en que las actividades productoras de objetos o de obras se reproducen ellas mismas. (Lefebvre, 1968:29)

Fiel a su tradición marxista, el autor no sólo hace una lectura de la vida cotidiana como reflejo de una estructura de dominación, sino que complementa esa idea con la potencialidad 
transformadora de "lo cotidiano". Asimismo, puede observarse que también rescata las ideas de producción y reproducción que se han abordado.

Aquí se entiende que el concepto de vida cotidiana tiene una implicancia clave en el entendimiento de la noción de derecho a la ciudad que el mismo autor trabaja a lo largo de su producción académica, y se vincula estrechamente con la idea de "prácticas urbanas", que se definirán como usos y sentidos que un grupo humano da a la ciudad y sus bienes en un momento histórico determinado.

Ahora bien, no debe entenderse que el concepto anteriormente brindado pretende universalizar, al estilo del liberalismo político clásico, la experiencia del grupo social dominante para constituirlo en molde de futuras políticas públicas destinadas a la ciudad como derecho. Para reconstruir las prácticas urbanas de un grupo humano determinado en un momento histórico determinado y desde una perspectiva de género, este artículo propone indagar en el enfoque elaborado por Dorothy Smith (2005), denominado por ella misma "Etnografía Institucional", y que busca explorar una "Sociología para la gente", es decir, (re)subjetivar a las personas que el cientista social se propone investigar y producir conocimiento que impacte en su vida cotidiana en lugar de tomarlos como meros objetos de estudio. Para ello, la autora elabora la estrategia conocida como "Women's standpoint [punto de vista de las mujeres]" por ser ellas sujetos epistemológicamente privilegiados ${ }^{4}$ para dar cuenta de las experiencias del día a día o, en la terminología lefebvriana, de la vida cotidiana.

Al incorporarse al mundo del trabajo, las mujeres se mueven, contradictoriamente, entre las esferas de producción y reproducción, si bien el mandato patriarcal indica que su labor predominante debe ser doméstica. Esta brecha en la pretendida división entre el espacio público y privado se traduce, como ya se ha mencionado, en la configuración de prácticas urbanas diferenciadas. Desde su rol, las mujeres pueden dar cuenta tanto de su propia cotidianeidad como de otros grupos desaventajados que dependen de sus cuidados (niños, niñas y adolescentes, personas con discapacidad, adultos y adultas mayores, etc.) y el cientista social que aborde esa experiencia puede reconstruir la prenombrada vida cotidiana no ya como un mero sumario de hitos rutinarios, sino como hechos políticos. La propia Smith clarifica cuál es el objetivo del marco metodológico que propone:

\footnotetext{
${ }^{4}$ Respecto a las mujeres como sujetos epistemológicamente privilegiados para dar cuenta de las "ruling relations [relaciones de gobierno]" a las que refiere Dorothy Smith se sugiere la lectura de los capítulos 1 y 2 de "Institutional etnography: A sociology for people".
} 
Aladro, A. Capitalismo, patriarcado y ciudad: generalidades de la producción de lo urbano desde una perspectiva de género. Derecho y Ciencias Sociales. Mayo- Octubre $2019 \mathrm{~N}^{\circ} 21$. (Derecho, ciudad y propiedad) Pgs 23-36. ISNN 1852-2971. Instituto de Cultura Jurídica y Maestría en Sociología Jurídica. FCJ y S. UNLP

Like a map, it aims to be through and through indexical to the local sites of people's experience, making visible how we are connected into the extended social relations of ruling and economy and their intersections. And though some of the work of inquiry must be technical, as mapmaking is, its product should be ordinarily accesible and usable, just as well-made maps is, to those on the terrain it maps [Al igual que un mapa, apunta a ser a través del índice de los sitios locales de la experiencia de las personas, haciendo visible cómo estamos conectados a la red extendida de las relaciones sociales de gobierno y economía y sus intersecciones. Y aunque parte del trabajo de indagación debe ser técnico, como lo es la cartografía, su producto deberá ser accesible y utilizable, al igual que los mapas bien hechos, para aquellos que están en el terreno que mapea]. (Smith, 2005:29)

A la vez que define su necesidad:

Institutional Etnography begins by locating a standpoint in an institutional order that provides the guiding perspective fromwich that the order will be explored. It begins with some issues, concerns, or problems that are real for people and that are situated in their relationships to an institutional order. Their concerns are explicated by the researcher in talking with them and thus set the direction of inquirí [La Etnografía Institucional inicia localizando un punto de vista en un orden institucional que provea una perspectiva guía desde la cual ese orden pueda ser explorado. Empieza con algunas complicaciones, preocupaciones o problemas que son reales para las personas y que están situados en sus relaciones con un orden institucional. Sus preocupaciones son explicadas por el investigador en sus charlas con ellas y ellas fijan la dirección de la pesquisa]. (Smtih, 2005:32)

El relevamiento de hechos de lo cotidiano que expone Smith como metodología para la producción de conocimiento situado, accesible y útil para mujeres en cuyas vidas se indaga se presenta como una estrategia de impacto para la producción de política pública local ${ }^{5}$ destinada a la generación de procesos de construcción de ciudad que busquen

\footnotetext{
${ }^{5}$ I mean by this simply that when it comes to knowing her way around in it, how things get done, where the bus stop for B-line bus is, at wich supermarket she can pick up both organic vegetables and lactate-reduced milk, and all the unspecifiables of her daily doings and the local conditions on wich she relies-when it comes to knowing
} 
Aladro, A. Capitalismo, patriarcado y ciudad: generalidades de la producción de lo urbano desde una perspectiva de género. Derecho y Ciencias Sociales. Mayo- Octubre $2019 \mathrm{~N}^{\circ} 21$. (Derecho, ciudad y propiedad) Pgs 23-36. ISNN 1852-2971. Instituto de Cultura Jurídica y Maestría en Sociología Jurídica. FCJ y S. UNLP

romper la falsa dicotomía "público-privado" sobre la cual los gobiernos de corte liberal diseñan y planifican lo urbano.

\section{Conclusión}

Este trabajo recogió inicialmente la tesis de Scholz sobre la división de la vida social en actividades productivas e improductivas para, posteriormente, vincular estas categorías con la construcción teórica de Carole Pateman en torno a la existencia de una dicotomía esfera pública-esfera privada que contribuye a la imposición de roles sociales basados en la jerarquía de género. A partir de la toma de postura sobre los vínculos entre patriarcado, capitalismo y espacialidad, pudo darse una relectura de la obra de Falú para dotar de contenido desde la Filosofía Política a las categorías que emplea en las ideas centrales sostenidas en su trabajo académico, lo cual sumó a la urbanidad al eje de análisis. Finalmente, aceptando la importancia de la vida cotidiana como hecho político en los términos de Henri Lefebvre, se propuso como metodología a la Etnografía Institucional trabajada por Dorothy Smith, en tanto permite dar cuenta, mediante el relevamiento de la cotidianeidad de las mujeres, del orden institucional y las relaciones políticas y económicas en las cuales se encuentran inmersas las personas en un tiempo y lugar históricamente determinados.

El derrotero descripto permite concluir en una primera instancia de análisis, que se pretenderá profundizar en escritos subsiguientes a esta publicación, que la persistencia de una tajante división espacial como la planteada por el liberalismo político clásico enquistada en el ámbito de la política pública local produce ciudades para ser habitadas privilegiadamente por varones, ya que el mismo proceso de producción de la ciudad enmarcado en la lógica del capitalismo patriarcal generará una mercancía acorde a la misma. Al entender dialécticamente las articulaciones entre capitalismo y patriarcado, se entiende que las ciudades como dimensión espacial de las relaciones sociales no quedan exentas de contradicciones, siendo una de ellas el

these matters, she is an expert. It is another matter altogether when it comes to the forms of organization that authenticate the organic status of the vegetables that brings the supermarket or the bus company into daily existance; or taht constitute the responsability of the streets, the side walks, the standards of waste disposal, and so on [Quiero decir con esto, simplemente, que cuando se trata de conocer su manera, cómo se hacen las cosas, dónde está la parada de autobús de la línea $\mathrm{B}$, en cuál supermercado ella puede conseguir tanto vegetales orgánicos como leche reducida en lactosa, y todos los aspectos inespecificables de sus actividades diarias y sus condiciones locales en las que se encuentra: cuando se trata de conocer estos asuntos, ella es una experta. Es otra cuestión en general cuando se trata de las formas de organización que autentican el carácter orgánico de las verduras que vende el supermercado o la existencia diaria de la compañía de; o las formas de organización que implican la responsabilidad de las calles, los paseos laterales, los estándares de eliminación de residuos, etc.]. (Smith, 2005:24) 
hecho de que, a través de la incorporación de las mujeres al mercado de trabajo, las mismas deban transitar entre actividad productiva/espacio público y actividad reproductiva/espacio privado, lo cual les exige, al mismo tiempo, prácticas urbanas propias para cumplir esta "demanda".

Desde estas consideraciones, comprender a la ciudad como derecho desde una perspectiva de género necesariamente debe partir de una desigualdad presente en lo que se ha llamado "dimensión de acceso" entre varones y mujeres, derivada de la producción capitalista y patriarcal de lo urbano. Esta inequidad en las condiciones de acceso al derecho a la ciudad es contraria al concepto de igualdad desarrollado a partir del Pacto de Derechos Económicos, Sociales y Culturales (1966), cuyo Comité en su Observación General No 16 (2005) señala que es obligación inmediata de los Estados parte integrar planes de acción que mejoren el acceso de las mujeres a los derechos que el instrumento contempla.

Finalmente, para el diseño de las políticas públicas exigidas internacionalmente para garantizar el acceso a los bienes en condiciones de igualdad por parte de las mujeres, se propone que el relevamiento de datos para diagnóstico de la situación en un tiempo y espacio determinados se realice a través de la recuperación de la vida cotidiana como hecho político y su estudio desde la Etnografía Institucional para comprenderla como inmersa en un orden institucional con sus especificidades. El estudio de la cotidianeidad desde el punto de vista de las mujeres tiene, además, la potencialidad de extender sus conclusiones a otros grupos vulnerables cuyo cuidado, históricamente, ha estado en sus manos (adultos y adultas mayores, personas con discapacidad, niños, niñas y adolescentes).

\section{Bibliografía}

Borja, J. (2001). La ciudad del deseo. En: Carrión, F. (ed.). La ciudad construida urbanismo en América Latina (pp. 391-396). Quito: FLACSO.

Carlos, A. F. A. (2008). De la "geografía de la acumulación" a la "geografía de la reproducción": un diálogo con Harvey. Scripta Nova Revista Electrónica De Geografía $Y$ Ciencias Sociales [en línea), Vol. XII, núm. 270 (143). Disponible en: http://www.ub.edu/geocrit/sn/sn-270/sn-270-143.htm Engels, F. [1891] (2011). El origen de la familia, la propiedad privada y el Estado. La Plata: De la Campana. 
Falú, A. (1998), “Gestión democrática y descentralizada del territorio”. En: Tercera Cumbre de Mercociudades (pp.15-24). Córdoba: Comisión Municipal de Estudios y Asesoramiento para Igualdad de Oportunidades de la Mujer

Falú, A. (2016), "La omisión de género en el pensamiento de las ciudades”. En: Borja, J., Carrión, F. y Corti, M. (Eds.). Ciudades para cambiar la vida: Una respuesta a Hábitat III (pp. 159-169). Buenos Aires: Editorial Café de las Ciudades.

Federici, S. (2015), Calibán y la bruja: cuerpo y acumulación originaria. Ciudad Autónoma de Buenos Aires: Tinta Limón.

Harvey, D. (2007), “La geografía de la acumulación capitalista: una reconstrucción de la teoría marxista”. En: Espacios del capital: hacia una geografia crítica (pp. 255-284). Madrid: Ediciones Akal.

Harvey, D. (2012). Ciudades rebeldes: Del derecho de la ciudad a la revolución urbana. Madrid: Ed. Akal.

Jirón, P. y Zunino Singh, D. (2017). Dossier. Movilidad y Género: experiencias latinoamericanas. Revista Transporte y Territorio, (16), 1-8.

Lefebvre, H. (1967). El derecho a la ciudad. Barcelona: Península.

Lefebvre, H. (1968). La vida cotidiana en el mundo moderno. Madrid: Alianza Editorial.

Lefebvre, H. (1970). "Introducción a la psicosociología de la vida cotidiana". En: De lo rural a lo urbano (pp. 85-102). Barcelona: Ediciones Península.

Lefebvre, H. (1991). Critique of Everyday Life Volume I: Introduction. London: Verso [edición original (1947) Critique de la vie quotidienne I: Introduction, Paris: Grasset].

Lindón Villoría, A. (2004). Las huellas de Lefebvre sobre la vida cotidiana. Revista Veredas, 5(8), 39-60.

Lombardo, J. D. (2012). La construcción del espacio urbano: Sus características en el Área Metropolitana de Buenos Aires. Los Polvorines: Ediciones CICCUS.

Pateman, C. (1995). El contrato sexual. Buenos Aires: Antrophos.

Pateman, C. (2009), Críticas feministas a la dicotomía público/privado. En: Ávila Santamaría, R., Salgado, J. y Valladares, L. (Comp.). El género en el derecho. Ensayos críticos (pp. 37-68). Quito: Ministerio de Justicia y Derechos Humanos.

Scholz, R. (2013). El patriarcado productor de mercancías. Tesis sobre capitalismo y relaciones de género. Constelaciones. Revista de Teoría Crítica, (5), 44-60. 
Aladro, A. Capitalismo, patriarcado y ciudad: generalidades de la producción de lo urbano desde una perspectiva de género. Derecho y Ciencias Sociales. Mayo- Octubre $2019 \mathrm{~N}^{\circ} 21$. (Derecho, ciudad y propiedad) Pgs 23-36. ISNN 1852-2971. Instituto de Cultura Jurídica y Maestría en Sociología Jurídica. FCJ y S. UNLP

Sen, G. (1996). Una economía alternativa desde una perspectiva de género. En: Osch, T. V. (Ed.). Nuevos enfoques económicos: contribuciones al debate sobre género y economía (pp. 53-63). San José, Costa Rica: Embajada Real de los Países Bajos.

Smith, D. (2005). Institutional ethnography: A sociology for people. Toronto: Rowman Altamira.

Soja, E. (2014). En busca de la justicia espacial. Valencia: Tirant Humanidades. 